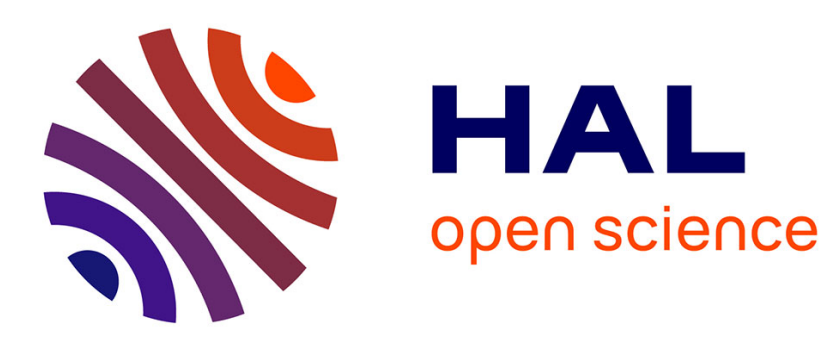

\title{
Tentative d'identification des productions métalliques des bronzes coulés dits " à la grosse tête "
}

Anne Geiser, Jean-Noël Barrandon

\section{To cite this version:}

Anne Geiser, Jean-Noël Barrandon. Tentative d'identification des productions métalliques des bronzes coulés dits " à la grosse tête ". Gallia - Archéologie de la France antique, 1995, Les potins gaulois, 52, pp.21-26. 10.3406/galia.1995.3117 . hal-01911266

\section{HAL Id: hal-01911266 \\ https://hal.science/hal-01911266}

Submitted on 7 Feb 2020

HAL is a multi-disciplinary open access archive for the deposit and dissemination of scientific research documents, whether they are published or not. The documents may come from teaching and research institutions in France or abroad, or from public or private research centers.
L'archive ouverte pluridisciplinaire HAL, est destinée au dépôt et à la diffusion de documents scientifiques de niveau recherche, publiés ou non, émanant des établissements d'enseignement et de recherche français ou étrangers, des laboratoires publics ou privés.

\section{(ㅇ)(1) $\$$}

Distributed under a Creative Commons Attribution - NonCommercial - NoDerivatives $\mid 4.0$ 


\title{
2.2. Tentative d'identification des productions métalliques des bronzes coulés dits «à la grosse tête »
}

\author{
Anne Geiser et Jean-Noël Barrandon ${ }^{5}$
}

\begin{abstract}
Résumé. Vers 1890, on a trouvé sur le col du Grand-Saint-Bermard, plus de 500 monnaies gauloises appartenant à un dépôt votif. Le faciès des émissions compte $45 \%$ de potins, près de $50 \%$ de bronzes frappés à LT D2 et $6 \%$ de monnaies de Marseille et leurs imitations directes. Parmi vingt variétés de potins, ceux "à la grosse lête " représentent près de $40 \%$ des trouvailles de potin. Il est possible de subdiviser les potins "à la grosse tête ", type A, en plusieurs courants typologiques. Or la plupart des séries typologiques du potin "à la grosse tête " présentes en Suisse occidentale, figurent au Crrand-Saint-Bernard. Pour les subdiviser en classes cohérentes, plusieurs problèmes inhérents à l'étude des potins se posent. Les critères de classification des potins ne peuvent pas être les mêmes que ceux des monnayages frappés. Chaque production de polins est en principe obtenue par coulée de métal dans des moules en chapelets. Pour tenter un classement des potins "à la grosse lête ", type A, une analyse précise du contenu métallique des séries typologiques, devrail théoriquement permettre de. retrouver quelques ensembles cohérents. Ainsi, pourrait-on reconstruire au mieux, une arborescence de chapelets ou, plus largemenl, plusieurs productions réalisées avec un alliage plus ou moins semblable. Ces analyses globales ont aussi pour objectif d'évaluer la composition métallique des potins "à la grosse tête » avec justesse et de voir s’il y a une certaine corrélation entre composition et typologie.
\end{abstract}

Zusammenfassung. Fs ist möglich, die Potinmünzen " à la grosse tête » des Typrus A noch weiter in verschiedene gängige typologische Klassen aufzuteilen. Bei deren Unterteilung in kohärente Klassen treten einige, der Potinmünzenstudie inhärente Probleme auf. Die Klassifizierungskriterien der gegossenen Potinmünzen müssen sich von denen der gepräglen Münzen unterscheiden.

Im Prinzip wird bei der Potinproduktion durch melallguss in Multiformen vorgegangen. Unternimmt man den Versuch, den Typus A der Potinmünzen "à la grosse tête " zu klassieren, drängt sich eine genaue Analyse des Metallgehaltes der typologischen Serien auf, die es theoretisch erlauben sollte, einige kohärente Einheiten festzustellen. Somit könnte man aufs Besle sowohl eine Gussform oder auch einige Produktionen ähnlicher Legierungen nachbilden. Diese globalen Untersuchungen sollen auch dazu dienen, die Legierungen der Potinmünzen "à la grosse tête" mit Genauigkeit zu analysieren und damit festzuslellen, ob eine Relation zwischen der Legierung und der Typologie besteht.

Qu'appelle-t-on en réalité " potin »? Quels alliages et quels procédés utilisaient les Celtes pour fabriquer ces pièces? Est-il possible de reconnaître certains cnsembles métalliques au sein des séries typologiques à partir des comparaisons d'alliages et ainsi de définir une ou plusieurs productions métalliques?

Pour tenter de répondre à ces questions, nous avons choisi d'étudier le potin dont la dispersion et le nombre de types, sont parmi les plus importants : le potin dit « à la grosse tête ».

\section{Problème DE la ClaSSIFICATION DES BRONZES COULÉS}

Les potins « à la grosse tête » de la série A se répartissent en plusieurs courants typologiques différents et, semble-t-

$\overline{5 . ~ L e s ~ a u t e u r s ~ r e m e r c i e n t ~ p a r t i c u l i e ̀ r e m e n t ~ C l a u d e ~ B r e n o t ~ p o u r ~ s e s ~}$ conseils avisés au début de cette recherche et le personnel du cyclotron d'Orléans (CERI du CNRS) pour les facilités d'irradiation. il, cohérents ${ }^{6}$. Les types peuvent être subdivisés selon la dimension des flans, selon la présence ou l'absence de filets périphériques et selon l'importance de la tête du taureau au revers : types $\mathrm{A} 1$ (variantes $\mathrm{Al}, 1, \mathrm{~A} 1,2, \mathrm{A1}, 3$ ), A2, (variantes A2,1-4) A3 (variantes A3,1-3) et A5 (A5,1-2).

Dans le cas de l'or, de l'argent ou du bronze frappé, l'identification et la comparaison des coins monétaires et de leurs liaisons, permettent la subdivision des monnayages en classes et, parfois avec des études de métrologie et de titre, la reconstitution de la chronologie relative des émissions.

Pour parvenir à identifier et à reconstruire les différentes productions, outre la sériation typologique et l'examen de la dispersion des types, un certain nombre de problèmes se posent, inhérents au procédé de fabrication des bronzes coulés.

6. A. GeISER, K GRURL, Essai de typologie des potins "à la grosse tête ", Gazette Numismatique Suisse, 43/170, 1993, p. 25-35 et ci-dessus en 2,1. 


\section{LA FABRICATION DU BRONZE COULÉ}

Cette fabrication ne nécessite qu'une seule opération. Ces pièces étaient coulées dans des moules à alvéoles communicants (technique de coulée "en chapelet "). L'artisan avait préalablement imprimé ou gravé les types à représenter dans les alvéoles, soit probablement à l'aide de matrices en relief, soit par "surmoulage " avec des pièces déjà existantes ${ }^{7}$. Dans certains cas, il n'est pas exclu que plusieurs « chapelets » aient été reliés entre eux ${ }^{8}$.

L'identification de ces matrices ou coins à partir des potins est difficile, voire impossible en raison de la technique même, offrant aux reliefs des contours vagues et imprécis. La métrologie globale des potins « à la grosse tête " est de surcroît très variable ${ }^{9}$. On ne parvenait probablement pas à régler exactement les dimensions des moules (profondeur et diamètre) ${ }^{10}$.

L'identification des moules et de la métrologie des bronzes coulés ne sont pas de grande utilité pour établir la chronologie relative de ceux-ci.

Les critères de classification des bronzes coulés ne sont donc pas les mêmes que ceux des monnayages frappés.

\section{CRITÈRES TECHNIQUES DE CLASSIFICATION}

Il fallait trouver d'autres critères de classification à ajouter à ceux dont nous disposions pour parvenir à identifier et

7. Pour la fabrication des potins on se rapportera aux articles de $M$. THIRION, I e trésor de Fraire : monnaies gauloises en potin, $R B N, 108$, 1962, p. $72-78$ et J.-B. COLBERT DE BEAULIEU, Les monnaies gauloises coulées, in : J.-M. Dentzer, Ph. Gauthier et T. Hackens (éd.), Numismatique antique : Problèmes et méthodes, Acles du colloque organisé à Nancy du 27 septembre au 2 octobre 1971, Nancy-Louvain, 1975, p. 23-26 (Annales de l'Est, 44).

8. M. THIRION, op. cit, 1962, p. 77 , illustre des pièces coulées encore reliées entre elles et issues d'un moule en forme "d'arbre " (ensemble pour fabriquer des "fulus " marocains en 1845 conservé au British Museum).

9. J.-B. COLBERT DE BEAULIEU, op. cit., 1975, p. 26, relève des variations de poids allant du simple au triple.

10. L'examen des potins " à la grosse tête "A représentés au GrandSaint-Bernard montre néanmoins que $: 1$, le poids moyen des pièces varie généralement en fonction des diamètres; 2 , les diamètres de plusieurs séries typologiques prises séparément sont ajustés, par conséquent le poids des pièces devrait l'être aussi! Cette piste est à explorer plus avant. Il convient de noter aussi que la non homogénéité pondérale est également un phénomène propre aux monnayages de bronze, en particulier pour les bronzes dont les flans étaient coulés dans des moules avant la frappe : l'as de la République romaine ou le bronze de Marseille par exemple. Les variations de poids relevées sont également le résultat de la variation de la composition des alliages, de l'usure et des corrosions subies par ceux-ci au cours des ans. éventuellement reconstruire les productions. De la méthode de classement traditionnel appliquée aux monnayages frappés, subsiste l'examen de la composition de l'alliage.

Le principe de la production des bronzes coulés se caractérise par une seule fonte de métal, donc un alliage plus ou moins comparable par émission de chapelet ou "d'arbre de chapelets" ${ }^{11}$. Il est donc nécessaire de connaître l'alliage d'un échantillon important de potins de mêmes types pour parvenir à retrouver ces fontes ou émissions d'origine. En raison du nombre de types différents connus dans le cas des potins « à la grosse tête ", cette opération paraît statistiquement difficile. Elle devrait théoriquement être possible dans le cas d'émissions dont la dispersion est géographiquement proche de la production.

L'examen de l'alliage devrait également permettre de déterminer la composition et la précision de " réglage " des différents alliages entre eux.

\section{ESTIMATION DU NOMBRE DE POTINS PAR CHAPELET}

Pour parvenir à identifier des séries d'alliages, il faut savoir combien de potins étaient fabriqués par fonte de métal.

Comme l'a montré Thirion pour les potins " au ramcau ", la préscncc sur un ccrtain nombrc de potins de deux jets ou tenons de coulée, placés à $180^{\circ}$ l'un de l'autre, indique que la fonte était effectuée dans des moules à alvéoles communicants, placés dans le prolongement les uns des autres ${ }^{12}$.

Il propose une méthode statistique pour évaluer le nombre d'alvéoles que pouvait compter un chapelet et ainsi parvenir à estimer le nombre de pièces fabriquées par fonte de métal. Il s'agit simplement de relever le nombre de tenons par potin et à partir du rapport statistique obtenu de calculer le nombre possible d'alvéoles par chapelet.

L'examen des tenons de coulée d'environ 150 potins « à la grosse tête » de la série A, montre que pour $100 \%$ des individus qui présentent deux tenons, ceux-ci sont placés à $180^{\circ}$ l'un de l'autre quels qu'en soient les types. Cela signifie que nous sommes en présence de monnaies coulées en chapelets longilignes. Ce qui n'exclut pas tou-

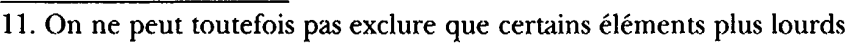
entrant dans la composition des alliages, comme le plomb, se soient déposés de façon plus importante dans les alvéoles les plus basses du chapelet au moment de la coulée.

12. M. THIRION, op. cit., 1962, p. 77. 
tefois la possibilité de plusieurs chapelets reliés entre eux sous forme d'arbre.

Sur cent cinquante potins "à la grosse tête " $\mathrm{A}$, examinés, près de $15 \%$ présentent un seul tenon de coulée. Il fallait donc probablement des moules avec au moins dix alvéoles par chapelet réunis ou non à d'autres, pour effectuer une coulée ${ }^{13}$. Chaque production de potins obtenue avec une seule fonte de métal comportait au moins dix pièces.

Pour tenter de reconstruire des ensembles de potins «à la grosse tête », type A cohérents, produits éventuellement dans un même chapelet, voire un même arbre ou, le cas échéant, avec un alliage plus ou moins semblable, nous avons effectué une analyse métallique précise de plusieurs individus des séries typologiques provenant d'une région donnée, la Suissc romande ${ }^{14}$.

\section{JUSTIFICATION DU CHOIX D'UN ÉCHANTILLON}

La plupart des séries typologiques de potins "à la grosse tête " A que l'on retrouve sur le territoire de la Suisse occidentale, figurent au Grand-Saint-Bernard ${ }^{15}$. Nous avons donc choisi quarante-sept potins « à la grosse tête » $\mathrm{A} d u$ Grand-Saint-Bernard pour mener à bien cette recherche. Pour nous assurer également de la qualité de la méthode, quelle quc soit la corrosion des pièces, neuf potins du site éponyme de La Tène, quatre de l'oppidum La Tène D1 du mont Vully et six de plusieurs sites du canton de Vaud ont été réunis également selon les critères de la typologie présentée ${ }^{16}$.

\section{MÉTHODE D'ANALYSE}

C'est une méthode nucléaire, l'analyse par activation avec les neutrons rapides de cyclotron que nous avons utilisée pour étudier la composition de ces pièces en alliage « cuivreux ». Nous rappellerons que cette méthode permet une analyse globale et non destructive; par simple spec-

13. Pour compléter cette analyse statistique basée sur un échantillon réduit à 150 individus et pour nous assurer d'un résultat encore plus large, nous nous proposons d'élargir notre échantillon aux pièces du Musée national suisse.

14. Séries typologiques de Suisse romande : A1 à A8. Voir A. GFISER, K. GRUEL, op. cil, 1993, p. 30-32.

15. A. GEISER, K. GRUFL, op. cit., 1993, p. 30-32 : Al-A8.

16. Nous remercions Marguerite Spoerri du Cabinet de numismatique, musée d'Art et d'Histoire de Neuchâtel pour nous avoir prêté les pièces de La Tène et Anne-Francine Auberson du Service arrhéologique cantonal de Fribourg, pour les pièces du mont Vully. trométrie après irradiation, ce sont dix éléments $(\mathrm{Cu}$, $\mathrm{Sn}, \mathrm{Pb}, \mathrm{Sb}, \mathrm{As}, \mathrm{Ni}, \mathrm{Fe}, \mathrm{Ag}, \mathrm{Au}, \mathrm{Zn}$ ) qui sont ainsi dosés jusqu'à l'échelle de ppm $(10-6 \mathrm{~g} / \mathrm{g}){ }^{17}$. On notera que dans le cas des potins, le zinc est inférieur à la limite de détection $(<100 \mathrm{ppm})$ et que la valeur du fer n'est pas donnée car elle fluctue beaucoup et provient en grande partie de la gangue en terre.

\section{RÉSULTATS}

Les résultats obtenus sur les quatre-vingt potins analysés sont donnés dans le tableau ci-joint (tabl. 2, p. 26). D'un point de vue analytique, ils montrent la pertinence de la méthode utilisée : en effet, des pièces de même type mais de provenances différentes, donc dc modcs de conservation très différents, donnent des résultats identiques; c'est le cas, par exemple, du type A3,1 dont les pièces proviennent de quatre sites (Grand-Saint-Bernard, Fiex, La Tène et Lomont) et dont les résultats sont tout à fait comparables et dans les marges d'erreurs de la méthode d'analyse $(\mathrm{Sn}=21,2 \pm 2)$. Ils montrent que, si l'on excepte une pièce qui est en fer ( $n^{\circ} 83$ du site de La Tène de type $\left.A 4,2\right)$, toutes les autres sont des bronzes (cuivre et étain) contenant des proportions variables de plomb (de 0,01 à $30 \%$ ).

Si l'on trace le graphique en échelle logarithmique de la teneur en plomb en fonction de celle en étain (fig. 7), il apparait de façon très nette que trois types d'alliages ont été utilisés pour fabriquer ces potins :

1 , un bronze sans plomb $(\mathrm{Pb}<0,05 \%)$ contenant environ $20 \%$ d'étain;

2, un bronze contenant des traces de plomb $(0,1 \%$ $<\mathrm{Pb}<3 \%$ ) et environ $20 \%$ d'étain;

3 , un bronze au plomb; le plomb $(3 \%<\mathrm{Pb}<30 \%)$ se substituant à l'étain qui, de ce fait, diminue dans l'alliage.

Voyons maintenant comment se répartissent les différents types étudiés dans les trois alliages précédemment définis.

Le tableau 3 (p. 26) résume les différentes attributions.

La concordance, type-alliage, est tout à fait bonne pour les types A5, A6 et A8; on notera que les A5 et A8 sont produits avec le même alliage, ce qui peut indiquer une localisation unique de la fabrication ou (et) une production contemporaine.

17. F. BEAUCHESNE., J.-N. BARRANDON, Méthode d'analyse globale et non destructive du $\mathrm{Cu}$ et de ses alliages par activation avec des neutrons rapides de cyclotron, Revue d'Archéométrie, 10, 1986, p. 75-85; M.-F. GUERRA et alii, Ion Beam Analysis of Copper and Copper Alloy Coins, Archaeometry, 1988, 30/II. 
$\mathrm{Pb}$

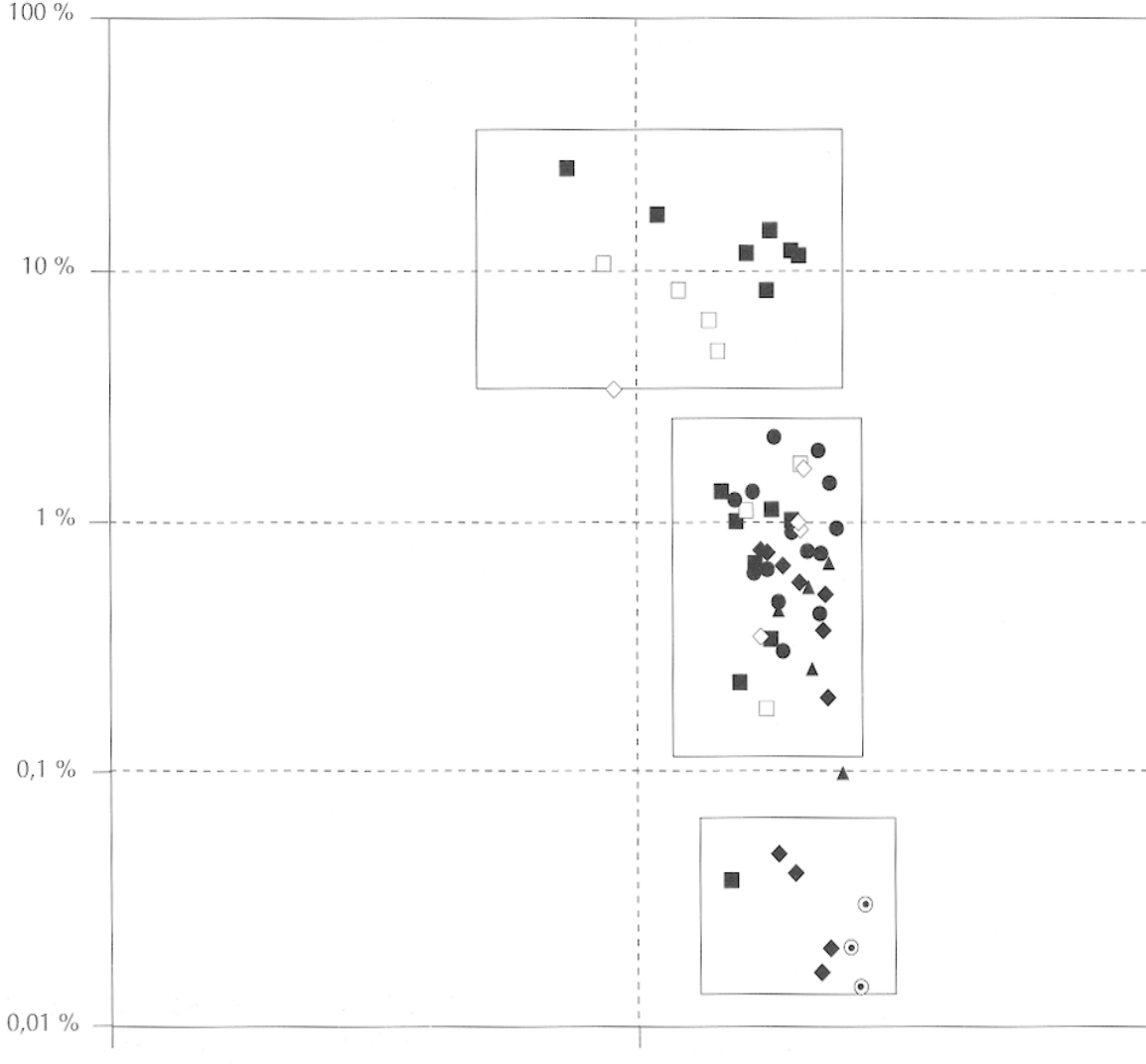

- $\mathrm{A} 1$

$\square \mathrm{A} 2$

$\diamond \mathrm{A} 3$

$\checkmark$ A4

$\triangle \mathrm{A} 5$

- A6

- A8

Fig. 7. Teneur en plomb en fonction de celle en étain dans les potins analysés (échelle : log.-log.).

$1 \%$

$10 \%$

$100 \%$ Sn

$\mathrm{Pb}$

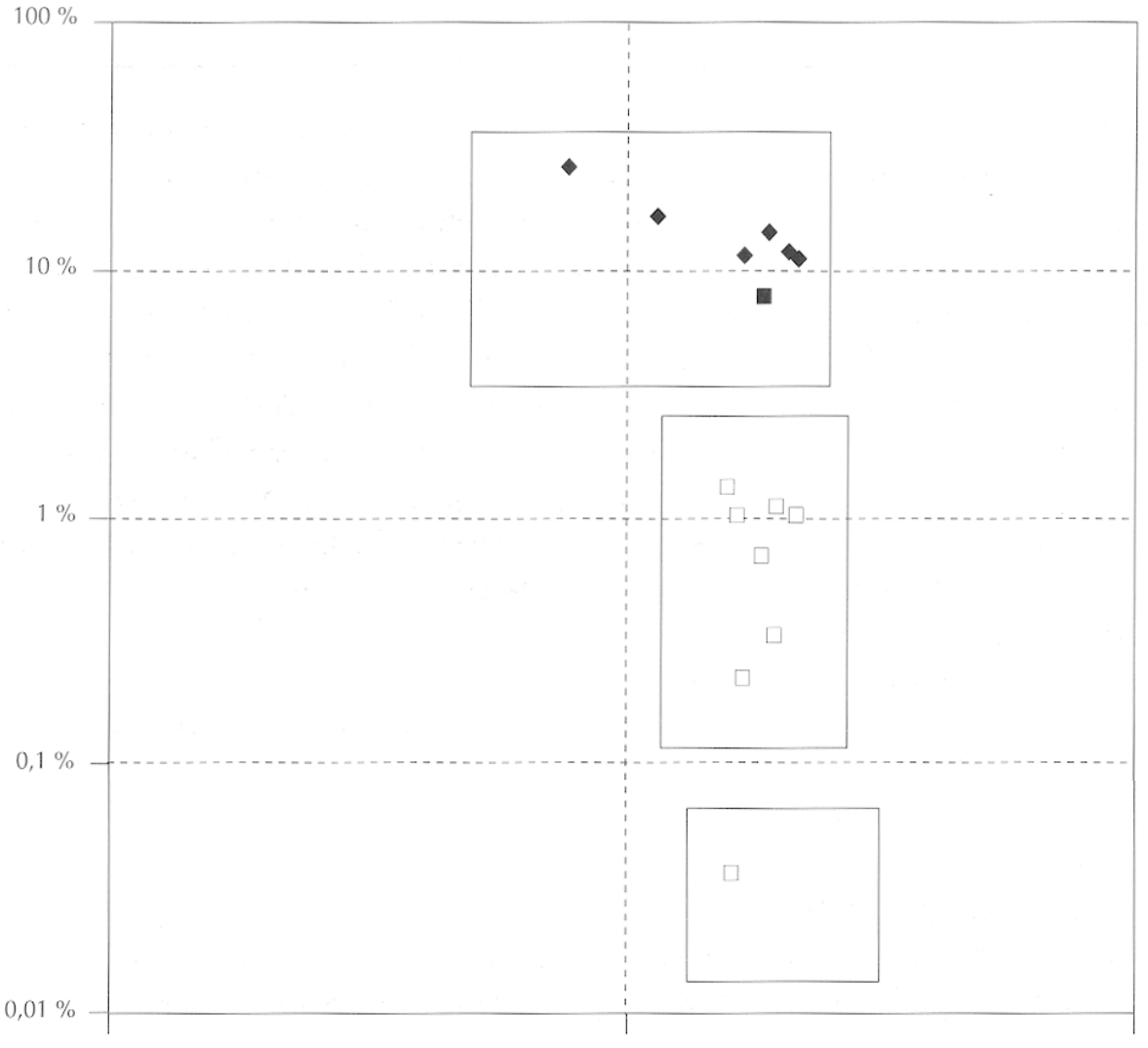

미, 1

$\square \quad \mathrm{A} 1,2$

- A1,3

Fig. 8. Teneur en plomb en fonction de celle en étain potins de type AI (échelle : $\log$ - $-\log$.). 


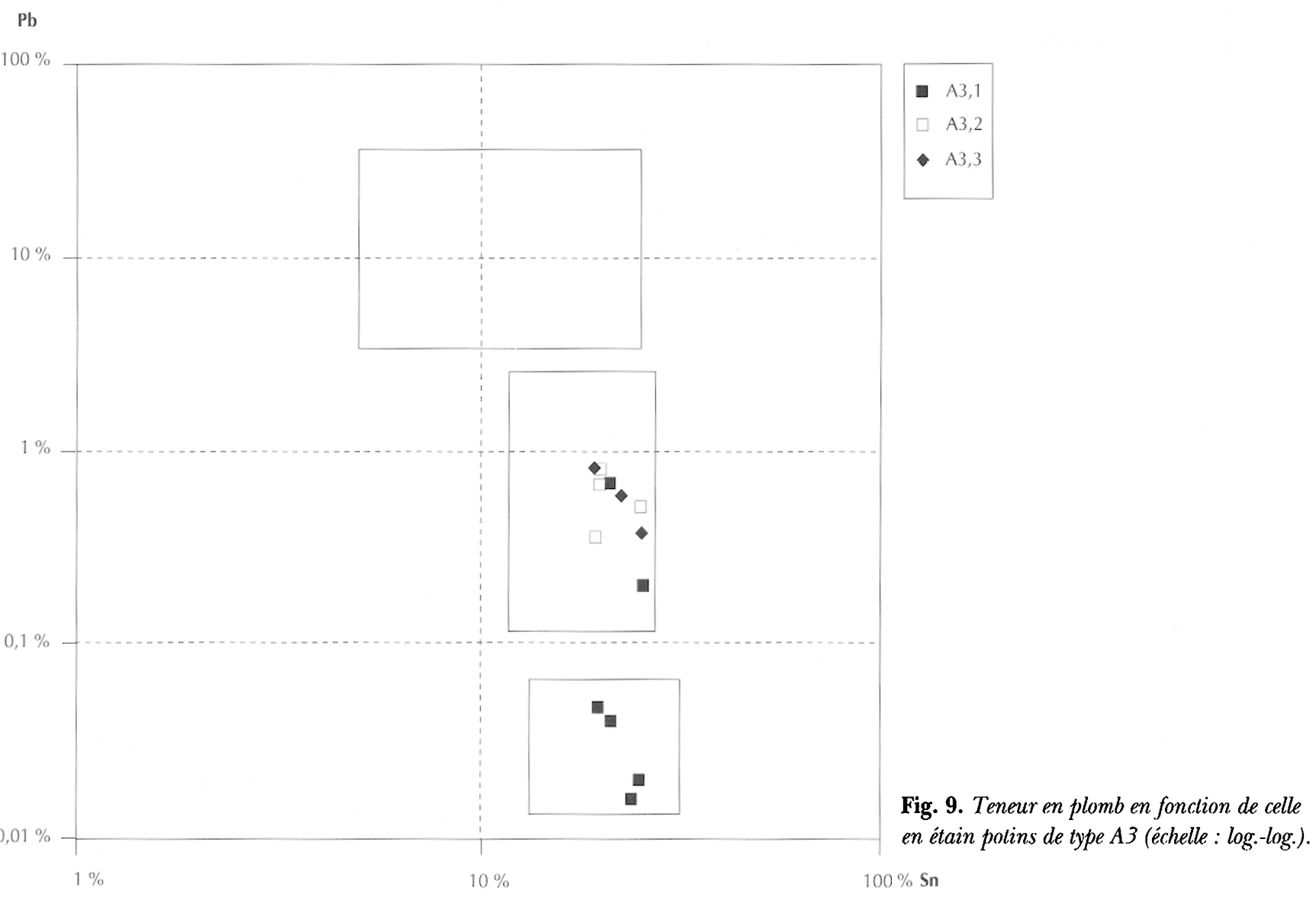

Pour le type A4, si l'on excepte une pièce $\left(\mathrm{n}^{\circ} 37 \mathrm{du}\right.$ Grand-Saint-Bernard), l'ensemble est fabriqué avec le même alliage 2 que les types 5 et 8 .

Par contre, les types A.3 et A1 se répartissent dans deux ou trois alliages différents. Si l'on fait une étude typologique plus fine des types $\mathrm{A} 1$ et $\mathrm{A} 3$, on se rend compte que ceux-ci peuvent se diviser en trois sous-types ou variantes. Étudions la répartition sous-type/alliages pour les deux types A1 et A3.

Les résultats (fig. 8 et 9 ) montrent que :

- pour le type $\mathrm{Al}$, les variantes $\mathrm{Al}, 1$ et $\mathrm{Al}, 3$ sont produites avec l'alliage 3 , tandis que la variante $A 1,2$ est coulée avec l'alliage 2 excepté pour une pièce $\left(\mathrm{n}^{\circ} 10\right)$ qui ne contient pas de $\mathrm{Pb}$;

- pour le type A3, les variantes A3,2 et A3,3 sont coulées avec l'alliage 2 tandis que la variante $\mathrm{A} 3,1$ est produite conjointement avec les alliages 1 et 2 .

En conclusion, les premières analyses des potins dits " à la grosse tête " montrent qu'il y a une assez bonne concordance entre la typologie et la nature de l'alliage; il est évident que ces premiers résultats demandent à être étoffés par d'autres analyses provenant d'autres sites, comme celui du mont Beuvray par exemple. Il faudra aussi tenter d'expliquer « le pourquoi et le comment » de ces productions. S'agit-il de productions simultanées dans différents centres? S'agit-il de productions successives dans un ou plusieurs centres? Autant de questions que la suite de nos recherches en matière d'analyses métalliques, mais aussi que les potins en contextes archéologiques et les études de répartitions géographiques des différents types ${ }^{18}$ contribueront à résoudre.

\section{Anne GEISER et Jean-Noël BARRANDON}

18. Les résultats sommaires de la répartition des potins " à la grosse tête " en Suisse occidentale sont exposés ci-dessous en 4,2. 
Tabl. 2. Résultats de l'analyse des potins (Barrandon, Geiser).

\begin{tabular}{|c|c|c|c|c|c|c|c|c|}
\hline No & $\mathrm{Cu}$ & Sn & $\mathrm{Pb}$ & $\mathrm{Sb}$ & As & $\mathrm{Ni}$ & $\mathrm{Ag}$ & $\mathrm{Au}$ \\
\hline \multicolumn{9}{|l|}{$\mathrm{A} 1-1$} \\
\hline 1 & 73,2 & 17,9 & 8,3 & 0,25 & 0,083 & 0,11 & 0,058 & 0,0012 \\
\hline \multicolumn{9}{|l|}{$\mathrm{A} 1-2$} \\
\hline 2 & 82,2 & 15,5 & 1 & 0,023 & 0,24 & 0,12 & 0,023 & 0,0027 \\
\hline 3 & 80,8 & 15,1 & 0,037 & 0,01 & 0,17 & 0,26 & 0,025 & 0,0028 \\
\hline 4 & 80,3 & 18,2 & 1,1 & 0,1 & 0,031 & 0,04 & 0,047 & 0,0008 \\
\hline 5 & 82,9 & 15,8 & 0,23 & 0,047 & 0,5 & 0,07 & 0,038 & 0,014 \\
\hline 6 & 77,7 & 20 & 1 & 0,58 & 0,33 & 0,1 & 0,12 & 0,0019 \\
\hline 7 & 83,4 & 14,6 & 1,3 & 0,027 & 0,27 & 0,1 & 0,018 & 0,0029 \\
\hline 9 & 81,1 & 17 & 0,69 & 0,022 & 0,25 & 0,12 & 0,019 & 0,0027 \\
\hline 10 & 79,9 & 18,2 & 0,34 & 0,43 & 0,85 & 0,031 & 0,22 & 0,019 \\
\hline \multicolumn{9}{|l|}{ A1-3 } \\
\hline 8 & 67,1 & 18,2 & 14,4 & 0,074 & 0,1 & 0,08 & 0,057 & 0,0012 \\
\hline 11 & 66 & 7,5 & 25,9 & 0,066 & 0,35 & 0,031 & 0,1 & 0,0014 \\
\hline 12 & 68 & 20 & 12 & 0,06 & 0,32 & 0,03 & 0,08 & 0,003 \\
\hline 13 & 67,4 & 20,75 & 11,3 & 0,06 & 0,27 & 0,032 & 0,086 & 0,0013 \\
\hline 14 & 71,5 & 11,1 & 16,7 & 0,11 & 0,33 & 0,063 & 0,07 & 0,0042 \\
\hline 80 & 70,5 & 16,4 & 11,7 & 0,27 & 0,69 & 0,061 & 0,081 & 0,0036 \\
\hline \multicolumn{9}{|l|}{$A 2-1$} \\
\hline 15 & 77,2 & 22,4 & 0,016 & 0,02 & 0,022 & 0,036 & 0,0048 & 0,0002 \\
\hline \multicolumn{9}{|l|}{ A $2-2$} \\
\hline 18 & 78,7 & 13,8 & 6,4 & 0,42 & 0,076 & 0,15 & 0,35 & 0,0008 \\
\hline 24 & 79 & 14,4 & 4,8 & 0,43 & 1 & 0,067 & 0,32 & 0,0003 \\
\hline \multicolumn{9}{|l|}{$\mathrm{A} 2-3$} \\
\hline 16 & 77,9 & 8,8 & 10,7 & 0,58 & 1,25 & 0,086 & 0,44 & 0,0006 \\
\hline 17 & 81,5 & 17,8 & 0,18 & 0,19 & 0,07 & 0,087 & 0,1 & 0,0005 \\
\hline 23 & 78 & 12,3 & 8,3 & 0,45 & 0,41 & 0,062 & 0,29 & 0,0003 \\
\hline \multicolumn{9}{|l|}{$A 2-4$} \\
\hline 21 & 76,5 & 20,6 & 1,64 & 0,47 & 0,25 & 0,06 & 0,45 & 0,0041 \\
\hline 22 & 81,9 & 16,2 & 1,11 & 0,21 & 0,037 & 0,27 & 0,41 & 0,0008 \\
\hline \multicolumn{9}{|l|}{ A3-1 } \\
\hline 52 & 77,3 & 22,5 & 0,016 & 0,033 & 0,019 & 0,071 & 0,038 & 0,0004 \\
\hline 32 & 80,9 & 18,6 & 0,047 & 0,078 & 0,02 & 0,15 & 0,048 & 0,0005 \\
\hline 33 & 79,3 & 19,1 & 0,66 & 0,36 & 0,057 & 0,13 & 0,19 & 0,0003 \\
\hline 34 & 76,2 & 23,3 & 0,2 & 0,031 & 0,018 & 0,1 & 0,035 & 0,0005 \\
\hline 78 & 78,9 & 20 & 0,04 & 0,28 & 0,036 & 0,011 & 0,049 & 0,0009 \\
\hline 79 & 76,2 & 23,5 & 0,02 & 0,063 & 0,013 & 0,16 & 0,034 & 0,0003 \\
\hline \multicolumn{9}{|l|}{$\mathrm{A} 3-2$} \\
\hline 31 & 80,2 & 18 & 0,76 & 0,55 & 0,023 & 0,11 & 0,26 & 0,00008 \\
\hline 27 & 80,4 & 17,6 & 0,36 & 0,81 & 0,038 & 0,14 & 0,34 & 0,0004 \\
\hline 29 & 79,9 & 17,9 & 0,64 & 0,9 & 0,079 & 0,21 & 0,17 & 0,0007 \\
\hline 82 & 75,2 & 22,8 & 0,51 & 0,93 & 0,17 & 0,065 & 0,32 & 0,0005 \\
\hline \multicolumn{9}{|l|}{ A3-3 } \\
\hline 19 & 74,6 & 22,8 & 0,37 & 1,11 & 0,54 & 0,05 & 0,32 & 0,0014 \\
\hline 28 & 80,8 & 17,5 & 0,78 & 0,51 & 0,022 & 0,1 & 0,25 & 0,0005 \\
\hline 30 & 77,7 & 20,5 & 0,57 & 0,65 & 0,041 & 0,12 & 0,25 & 0,0028 \\
\hline
\end{tabular}

\begin{tabular}{|c|c|c|c|c|c|c|c|c|}
\hline $\mathrm{N}^{0}$ & $\mathrm{Cu}$ & Sn & $\mathrm{Pb}$ & $\mathrm{Sb}$ & As & $\mathrm{Ni}$ & $\mathrm{Ag}$ & $\mathrm{Au}$ \\
\hline \multicolumn{9}{|l|}{$\mathrm{A} 4-1$} \\
\hline 20 & 76,6 & 20,9 & 1,59 & 0,38 & 0,011 & 0,037 & 0,15 & 0,0033 \\
\hline 37 & 78,8 & 9,2 & 3,4 & 6,7 & 0,8 & 0,23 & 0,84 & 0,005 \\
\hline \multicolumn{9}{|l|}{ A4-2 } \\
\hline 38 & 76,1 & 20,8 & 0,95 & 1 & 0,11 & 0,42 & 0,32 & 0,0004 \\
\hline 39 & 76,1 & 20,7 & 1 & 1,2 & 0,21 & 0,29 & 0,42 & 0,0007 \\
\hline 25 & 80,4 & 17,6 & 0,36 & 0,81 & 0,038 & 0,14 & 0,34 & 0,0004 \\
\hline \multicolumn{9}{|c|}{ A5-1 } \\
\hline 40 & 74,8 & 23,1 & 0,61 & 0,9 & 0,06 & 0,09 & 0,3 & 0,0006 \\
\hline 42 & 76,6 & 21,3 & 0,55 & 0,8 & 0,087 & 0,2 & 0,26 & 0,0005 \\
\hline 43 & 74,4 & 23,6 & 0,69 & 0,63 & 0,077 & 0,12 & 0,001 & 0,0005 \\
\hline \multicolumn{9}{|l|}{ A5-2 } \\
\hline 41 & 74,6 & 25 & 0,1 & 0,022 & 0,01 & 0,048 & 0,034 & 0,0008 \\
\hline 44 & 76,7 & 21,6 & 0,26 & 0,93 & 0,12 & 0,1 & 0,2 & 0,0016 \\
\hline 45 & 79,6 & 18,8 & 0,44 & 0,7 & 0,05 & 0,091 & 0,28 & 0,0006 \\
\hline \multicolumn{9}{|l|}{ A6-1 } \\
\hline 35 & 74,1 & 25,6 & 0,02 & 0,017 & 0,019 & 0,11 & 0,03 & 0,0003 \\
\hline 36 & 72,5 & 27,3 & 0,03 & 0,02 & 0,009 & 0,05 & 0,024 & 0,0002 \\
\hline 60 & 72,8 & 26,8 & 0,014 & 0,0025 & 0,008 & 0,013 & 0,006 & 0,0002 \\
\hline \multicolumn{9}{|c|}{$A 8-1$} \\
\hline 46 & 74,4 & 22,5 & 1,9 & 0,46 & 0,11 & 0,28 & 0,26 & 0,0025 \\
\hline \multicolumn{9}{|c|}{ A8-2 } \\
\hline 47 & 78,7 & 19,8 & 0,9 & 0,21 & 0,038 & 0,17 & 0,062 & 0,0003 \\
\hline 48 & 79,6 & 19,1 & 0,31 & 0,4 & 0,022 & 0,12 & 0,19 & 0,0001 \\
\hline 49 & 76,3 & 22,7 & 0,73 & 0,12 & 0,0077 & 0,014 & 0,11 & 0.0003 \\
\hline 50 & 79,2 & 17 & 0,63 & 1,28 & 0,01 & 1,53 & 0,29 & 0.0009 \\
\hline 51 & 77,7 & 21,2 & 0,74 & 0,05 & 0,0048 & 0,014 & 0,082 & 0,0004 \\
\hline 53 & 78,5 & 18,5 & 2,2 & 0,37 & 0,014 & 0,017 & 0,3 & 0,0005 \\
\hline 54 & 80,7 & 15,5 & 1,2 & 1,8 & 0,051 & 0,27 & 0,24 & 0,0013 \\
\hline 55 & 80,2 & 16,8 & 1,3 & 1 & 0,01 & 0,31 & 0,31 & 0,0007 \\
\hline 56 & 77 & 22,4 & 0,43 & 0,04 & 0,007 & 0,013 & 0,057 & 0,0002 \\
\hline 57 & 74,5 & 23,8 & 1,4 & 0,13 & 0,0043 & 0,018 & 0,12 & 0,0005 \\
\hline 58 & 79,1 & 18,8 & 0,48 & 0,95 & 0,1 & 0,035 & 0,18 & 0.0006 \\
\hline 59 & 74,2 & 24,5 & 0,93 & 0,11 & 0,0046 & 0,018 & 0,13 & 0,0002 \\
\hline 29 & 79,9 & 17,9 & 0,64 & 0,9 & 0,079 & 0,21 & 0,17 & 0,0007 \\
\hline
\end{tabular}

Tabl. 3. Attribution des alliages utilisés (Barrandon, Geiser).

\begin{tabular}{|c|c|}
\hline type & alliage utilisé \\
\hline A1 & $1-2-3$ \\
\hline A2 & $2-3$ \\
\hline A3 & $1-2$ \\
\hline A4 & $2-3$ \\
\hline A5 & 2 \\
\hline A6 & 1 \\
\hline A8 & 2 \\
\hline
\end{tabular}

